\title{
MoCapaci: Posture and gesture detection in loose garments using textile cables as capacitive antennas
}

\author{
Hymalai Bello, Bo Zhou, Sungho Suh, and Paul Lukowicz \\ DFKI and TU Kaiserslautern \\ Kaiserslautern, Germany
}

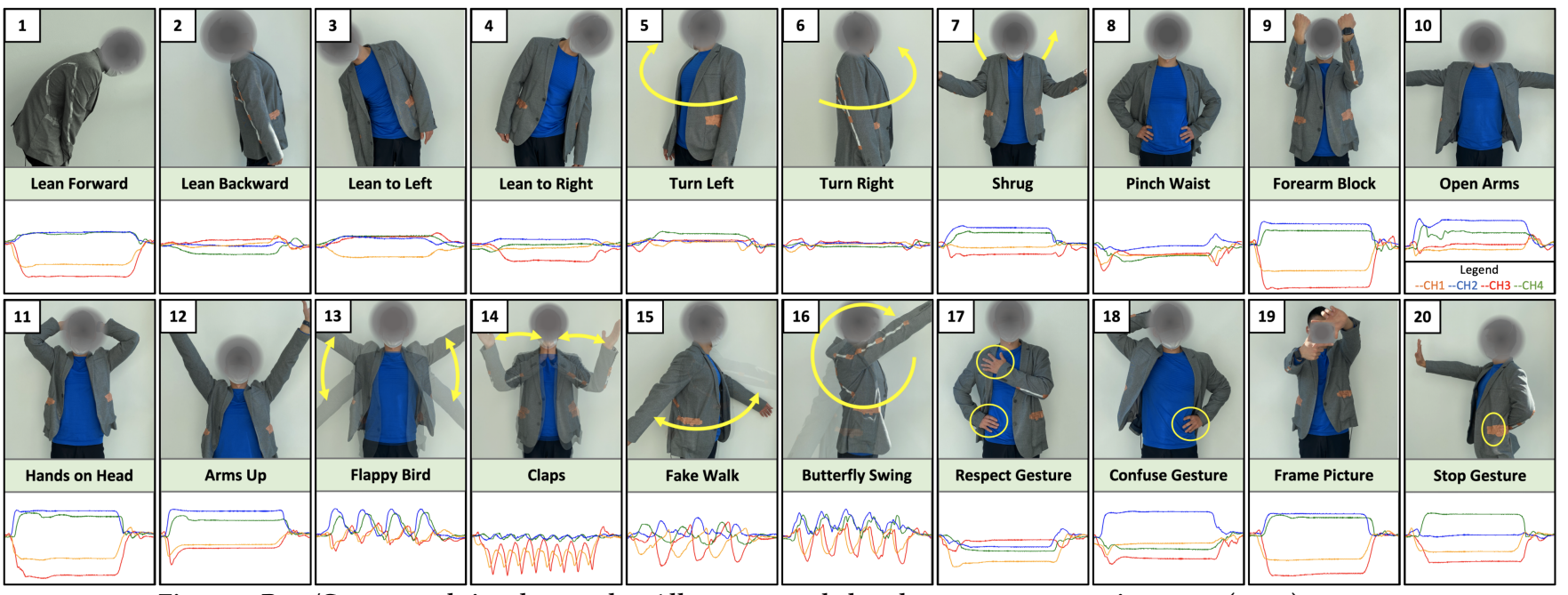

Figure 1: Pose/Gesture and signal examples. All axes are scaled to the same ranges: $x$ : time steps $(0,400)$, $y:$ norm

\begin{abstract}
We present a wearable system to detect body postures and gestures that does not require sensors to be firmly fixed to the body or integrated into a tight-fitting garment. The sensing system can be used in a loose piece of clothing such as a coat/blazer. It is based on the well-known theremin musical instrument, which we have unobtrusively integrated into a standard men's blazer using conductive textile antennas and OpenTheremin hardware as a prototype, the "MoCaBlazer." Fourteen participants with diverse body sizes and balanced gender distribution mimicked 20 arm/torso movements with the unbuttoned, single-sized blazer. State-of-the-art deep learning approaches were used to achieve average recognition accuracy results of $97.18 \%$ for leave one recording out and $86.25 \%$ for user independent recognition.
\end{abstract}

\section{CCS CONCEPTS}

- Computer systems organization $\rightarrow$ Embedded systems.

\section{KEYWORDS}

Loose garment sensing; capacitive sensing; theremin; activity recognition.

Permission to make digital or hard copies of all or part of this work for personal or classroom use is granted without fee provided that copies are not made or distributed for profit or commercial advantage and that copies bear this notice and the full citation on the first page. Copyrights for components of this work owned by others than ACM must be honored. Abstracting with credit is permitted. To copy otherwise, or republish, to post on servers or to redistribute to lists, requires prior specific permission and/or a fee. Request permissions from permissions@acm.org.

ISWC '21, September 21-26, 2021, Virtual, USA

(c) 2021 Association for Computing Machinery.

ACM ISBN 978-1-4503-8462-9/21/09 . \$ \$15.00

https://doi.org/10.1145/3460421.3480418

\section{ACM Reference Format:}

Hymalai Bello, Bo Zhou, Sungho Suh, and Paul Lukowicz. 2021. MoCapaci: Posture and gesture detection in loose garments using textile cables as capacitive antennas. In 2021 International Symposium on Wearable Computers (ISWC '21), September 21-26, 2021, Virtual, USA. ACM, New York, NY, USA, 6 pages. https://doi.org/10.1145/3460421.3480418

\section{INTRODUCTION}

Body postures and gestures (BPG) are key components of human activities, besides being essential ways to convey emotion and personality [22], implicit social interactions, sign language [15], etc. As a result, BPG recognition has been among the earliest wearable sensing applications, and today many mature, commercial applications (e.g., for motion capture) exist. Most popular wearable BPG sensing techniques use inertial measurement units (IMU) $[7,18,40]$ and, on the textile side, stretch sensors [10]. While highly effective in many applications, most current systems share one limitation: they require sensors to be firmly fixed to the body through tight garments or dedicated accessories, such as bracelets and straps. A reliable method for BPG recognition with loose garments remains a largely open problem. Existing approaches in loose-fitting wearables for BPG detection mostly have adopted complex sensing principles, as discussed in Section 2.1. This paper demonstrates a new simple method for BPG recognition with a loose garment based on non-contact capacitive sensing with off-the-shelf components. One potential use case is as a more sophisticated game controller for gesture-based games, such as the Nintendo Wii Rayman Raving Rabbids ®: TV Party - ShakeTV [54] or Just Dance ${ }^{\mathrm{TM}}$ [51]. Specifically, we adapt the well-known theremin musical instrument [45] to the purpose of BPG recognition. Typically a theremin consists of two long metal rod/loop antennas emitting sub-MHz frequencies. 
Table 1: Comparison with state-of-the-art sensing on garment methods for activity recognition.

\begin{tabular}{|c|c|c|c|c|c|}
\hline Studies & Device & Activities & Classification Method & Accuracy & Persons \\
\hline SMASH:Long sleeve shirt[18] & 3 accelerometers $(3 \mathrm{D})$ & 12 arm movements & Nearest Centroid Classifier & $95.00 \%$ & 8 \\
\hline Shirt:Digital Electronic[34] & Flexible fiber: 100 microchips with temperature sensing & 4 motor activities & $\mathrm{CNN}$ & $96.40 \%$ & 1 \\
\hline Jacket and pant [28] & Hetero-core fiber optics & 8 motor activities & SVM & $98.70 \%$ & 1 \\
\hline Sleeve [25] & Fabric-based triboelectric joint sensing & 4 daily activities & SVM & $91.30 \%$ & 14 \\
\hline RFID system [52] & 4 antennas; back, chest and feet & 5 motor +3 cleaning activities & SVM & $93.60 \%$ & 4 \\
\hline Sweat jacket [32] & Optical-strain sensor & 5 motor activities & CNN-LSTM & $90.90 \%$ & 12 \\
\hline Elastic sport band [62] & Textile pressure matrix (TPM) & 4 gym exercises +3 non-exercises & ConfAdaBoost & $93.30 \%$ & 6 \\
\hline Loose Pants [8] & Flexible piezoelectric & 5 motor activities +8 transitions & Rule-based algorithm [9] & $93.00 \%$ & 10 \\
\hline Trousers (3 sizes) [44] & Textile pressure sensors & 19 Sitting postures/gestures & Random Forest & $99.18 \%$ & 6 \\
\hline Air bladder band [21] & Air pressure sensors & 6 hand gestures & Fuzzy Logic & $90.00 \%$ & 6 \\
\hline Stretchable textile tape [39] & 8 nanocomposite pressure sensors & 10 American sign language numbers & $93.00 \%$ & 10 & \\
\hline Glove [41] & EGaIn-Silicone Soft: React to pressure or stretch & 12 Static hand gestures & Random Forest & $97.30 \%$ & 15 \\
\hline Leg/chest band, insole [17] & Capacitive & 5 motor activities & Bayesian Classifiers & $88.97 \%$ & 10 \\
\hline Our approach & Capacitive & 20 posture/gestures (Figure 1 ) & Conv2D & $97.18 \%$ & 14 \\
\hline
\end{tabular}

As the thereminist moves inside the antennas' range, volume and pitch can be controlled by him/her hand's position. We substituted the metal rod with soft wires and integrated them inside clothing.

In principle, any conductive wire/textile can be used as an antenna. Distinctive aspects in our design are a discrete gesture dictionary and the antennas move with the wearer's body motion, consequentially changing the signal. Our contributions include:

- Presenting a wearable approach for detecting BPG that does not require sensors to be firmly fixed to the body or integrated into a tight-fitting garment. Instead, sensing is incorporated into a loose fitting garment.

- Implementing a prototype, "MoCaBlazer" that adapts the famous theremin musical instrument [16] as a sensor merged into a loose man's jacket by integrating and modifying offthe-shelf components.

- Evaluating the proposed approach with the MoCaBlazer with 14 diverse participants in an experiment to detect 20 body postures and gestures.

- Applying several deep neural network models from the wearable HAR domain to the collected data, demonstrating accuracy of $86.25 \%$ for the leave-person-out (LPO) case and up to $97.18 \%$ for the leave-recording-out (LRO) scenario.

\section{RELATED WORK}

\subsection{Loose Fitting Wearables for BPG}

The use of IMU sensors distributed in a garment for BPG recognition is well-known approach $[7,18,40]$. EMG [60] has also been demonstrated as a viable alternative for distributed BPG sensing. Even though these systems provided accuracy above $90 \%$, they usually require the positions of the sensors to remain as stable as possible to reduce motion artifacts and noise. Furthermore, rigid sensors placed around the joints reduces the comfort of the user. One promising study employs 100 microchips with memory and temperature sensors interconnected in a flexible fiber on a T-shirt [34]. Strain-based sensing methods [4, 32, 35, 41] and pressure sensors $[21,35,39,41,44,62]$ have been proposed, but these depend on the stretchable properties of the garment. In the area of loose-fitting wearables, in a limited study [28], fiber optics were embedded in a jacket and pants, in which the wearer's movement would bend the optical fiber and cause changes in the transmitted light. Currently, optical fiber technology is evolving rapidly and multiple hardware designs have been developed [1, 27-29, 31, 50, 58]. A fabric-based triboelectric sleeve is proposed in Kiaghadi et al. [25]. Wang et al. [52] proposed four rigid RFID tags on the back, chest, and feet. Cha et al. [8] proposed four flexible piezoelectric sensors placed on the knee and the hip in slack pants.

Table 1 shows the comparison with a selection of past approaches. While most use complex sensing principles that cannot be easily integrated with e-textile components, our system uses commercial conductive textile parts as the key sensing antennas of the modified theremin, which is essentially capacitive sensing.

\subsection{Capacitive sensing}

Capacitive sensing $[6,57]$ is a well-explored sensing modality in pervasive and wearable computing for human activity recognition. The applications extend from capacitive furniture $[5,6,33,56]$, through capacitive wristbands [2, 3, 13, 38], rings [55], clothes $[19,43]$, collars $[11,12]$ and prosthesis [61] up to an entire wall painted as a capacitive array [59] for posture gesture detection.

Cheng et al. [12] validated a textile design of on-body capacitance measurement for a abroad set of activities such as eating, head inclinations, arms and leg movements; sensors were placed on the neck, wrist, upper leg, and forearm. However, this approach required the sensors to be fit snugly to the body. Singh et al. [43] proposed a flexible textile capacitive array placed on the upper-leg, with the aim of gesture recognition for patients with paralysis; they tested with five persons for swipe and hover gestures. In the area of posture recognition, a capacitive backpack [14] has been proposed and worn by eight volunteers as a receiver of electromagnetic (EM) noise from the power lines and electronic devices inside a room. This system achieved $93.00 \%$ accuracy for twelve different gestures: both-arms-up, left-arm-down, right-arm-down, both-arm-out-front, rotate arms, right-arm-wave, left-arm-wave, bend down, step right, step left, punch-twice-kick, and kick-punch-twice. These capacitive sensing studies $[12,14,43,59]$ with tightly coupled or stationary electrodes inspired us to use textile theremin antennas in loose garments for body posture and gesture detection.

\section{APPROACH AND DESIGN}

The theremin is an electronic musical instrument based on the frequency fluctuation of its antennas caused by the proximity with a person's hands. The human body could be modeled as a capacitor plate virtually connected to the earth and, in conjunction with the theremin's antennas (second plate), completes a capacitor [43]. Thus, human proximity changes the effective capacitance of the Clapp LC oscillator in Figure 2, affecting its frequency. A theremin has two antennas, one for volume (loop antenna) and another for pitch control (rod antenna) [45]. The idea behind our work is that different distances between body parts can describe different postures; thus, appropriately shaped antennas and embedded in garments will result in specific frequency profiles. 


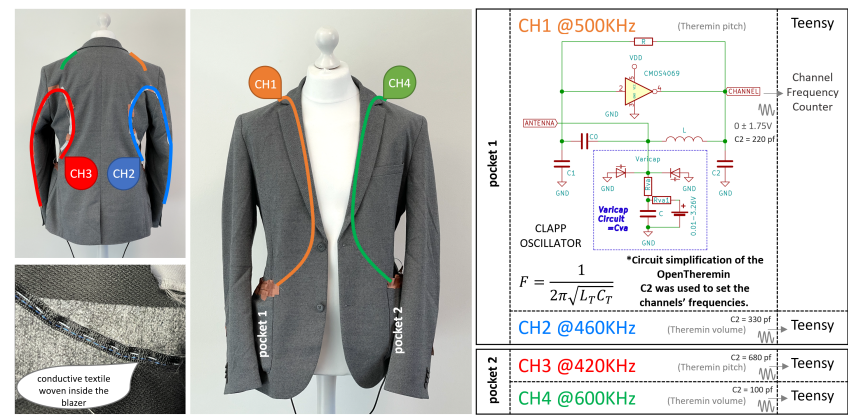

Figure 2: Smart MoCaBlazer Design

\subsection{Electronics and garment prototype}

We tested the approach mentioned above in a loose garment prototype, the MoCaBlazer, as shown in Figure 2. We designed four antennas to cover the chest, shoulders, back, and arms to detect upper body postures and gestures. As shown in Figure 2, the front antennas $(\mathrm{CH} 1, \mathrm{CH} 4)$ lead out of the pocket to the top button; then turn to align with the inner crease of the lapels till the notch; then lead out of the crease and climb around the shoulder to the back, and end at the middle edge of the shoulder pad. Textile cables (TWC24004B, Interactive Wear) [53] are used for the chest antennas and sewn directly on the canvas inside the lining with running stitches that do not pierce through the shell so that the antenna does not alter the structural design of the blazer. The back antennas $(\mathrm{CH} 2, \mathrm{CH} 3)$ come out of the pocket and run a curve over the latissimus dorsi muscles towards the deltoids; then turn sharply to go along the outer sleeve lines and end before the cuff buttons. As a blazer has many complex structures inside to keep its shape while the wearer can comfortably move, it is impracticable to sew the same textile cable inside the lining without hindering the wearer's freedom of motion in this particular design. Thus, we used fabric tapes to fix a standard 28 AWG electronic cable. The lengths of the antennas are $80 \mathrm{~cm}$ (front) and $100 \mathrm{~cm}$ (back) for this particular size (L/52). The theremin hardware is an OpenTheremin V3 [16]. In our design, two OpenTheremin boards are placed inside the blazer's pockets to support four channels. We altered the channel frequency by changing the capacitor (C2) in the clap-oscillator circuit to minimize cross-talk between channels as depicted in Figure 2. Using the Teensy ${ }^{\circledR} 4.1$ [49] development board, the data is sampled (frequency-count [48]) at $100 \mathrm{~Hz}$ per channel and sent by the serial port through USB to a computer, running a python data collection program.

\subsection{Experiment Design}

To test in an initial general dictionary and expand it in our future work for specific use-cases, we consider 20 body postures and gestures involving the upper body based on generic everyday movements as shown in Figure 1. Fourteen volunteers were asked to mimic these postures and gestures while standing and wearing the MoCaBlazer. The size L/52 blazer (Tom Tailor ${ }^{\circledR}$ ) is best suited for $184 \mathrm{~cm}$ tall persons. The experiment was divided into five sessions; each consists of four random repetitions per activity resulting in 400 instances per volunteer. Between each session, there was a rest time of 20 minutes on average. In some cases, the experiment was split over two days. The participants were seven women, 24-64 years old, and 157-183 cm in height; seven men, of 25-34 years old,

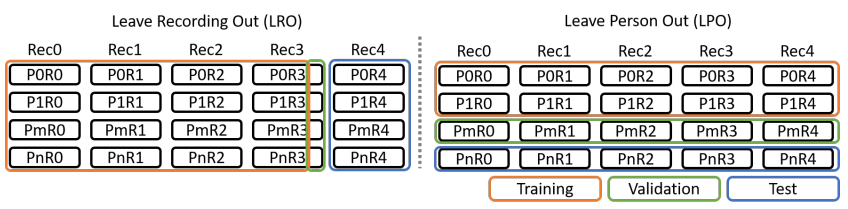

Figure 3: Train-valid-test schemes (P:person, R: recording)

Table 2: Comparison results (in \%) with various models.

\begin{tabular}{lllll}
\hline Method & Accuracy (LRO) & Accuracy (LPO) & Parameters & Training Time \\
\hline 1D-LeNet5 & $96.86 \pm 0.46$ & $85.34 \pm 7.83$ & 152,880 & $1.00 \mathrm{x}$ \\
DeepConvLSTM & $94.11 \pm 0.82$ & $85.42 \pm 5.84$ & 440,852 & $2.32 \mathrm{x}$ \\
Conv2D & $97.18 \pm 0.70$ & $86.25 \pm 8.09$ & 584,800 & $0.86 \mathrm{x}$
\end{tabular}

${ }^{1}$ LRO: leave recording out, LPO: leave person out.

2 The accuracy numbers are represented as mean $\pm s t d$, the standard deviation is from within each complete cross-validation.

${ }^{3}$ 1.00x Training time of 50min as baseline of complete LRO on NVidia RTX A6000 with the Tensorflow framework.

and $178-183 \mathrm{~cm}$ in height. The experiment was conducted in an office and without any calibration per user. All participants signed an agreement following the policies of the university's committee for the protection of human subjects. The experiment was video recorded for further confidential analysis. The observer and the participant followed an ethical/hygienic protocol according to the public health guidelines.

\section{EVALUATION}

The data is a time sequence of the frequency value generated by the Clapp oscillator of each channel, as it changed with the wearer's motion. The data of each gesture instance was filtered by a fourthorder Butterworth band-pass filtered from $1 \mathrm{~Hz}$ to $10 \mathrm{~Hz}$. Then the instance is normalized by subtracting the average of the first and last values of the gesture. The procedure is performed to eliminate the bias difference of the channels due to the fundamentals frequencies and remove the grounding dependency. Finally, the signal was resampled to 400 time-steps to provide a fixed input size for the deep learning model. ${ }^{1}$

\subsection{Data Analysis and Implementation Details}

The processed data of 14 volunteers (5600 instances) were fed to several deep learning models that have previously been shown to be effective in activity/gesture recognition such as modified 1DLeNet5 [30, 46], DeepConvLSTM [36], and Conv2D [23, 24, 42]. The modified 1D-LeNet5 model gives the best trade-off between performance, parameters, and training time as shown in Table 2. We defined the network as convolution (conv) - max pooling (maxpool)conv-maxpool-conv- fully connected (fc)-fc-softmax layers with batch normalization [20] and dropout [47] on the convolution layers. The input format is 400 timesteps by four channels. Leave recording out (LRO) and leave person out (LPO) paradigms are used as depicted in Figure 3, to see how well our method works in a known group of users, as well as with strangers. We ran all the person's permutations or recordings combinations within each run and summarized the confusion matrix together. That means a complete run of LRO has 5 and LPO has $14 \times 13$ train-valid-test cycles. The number of epochs used is 500, stopping when there are signs of overfitting. The three convolution layers are used with a kernel size of 41 and the activation function of ReLU. For max pooling, the pool size is $(40,40)$ for the first convolution $(400,40)$ and $(4,40)$

\footnotetext{
${ }^{1}$ https://github.com/drbz-zhou/MoCapaci
} 

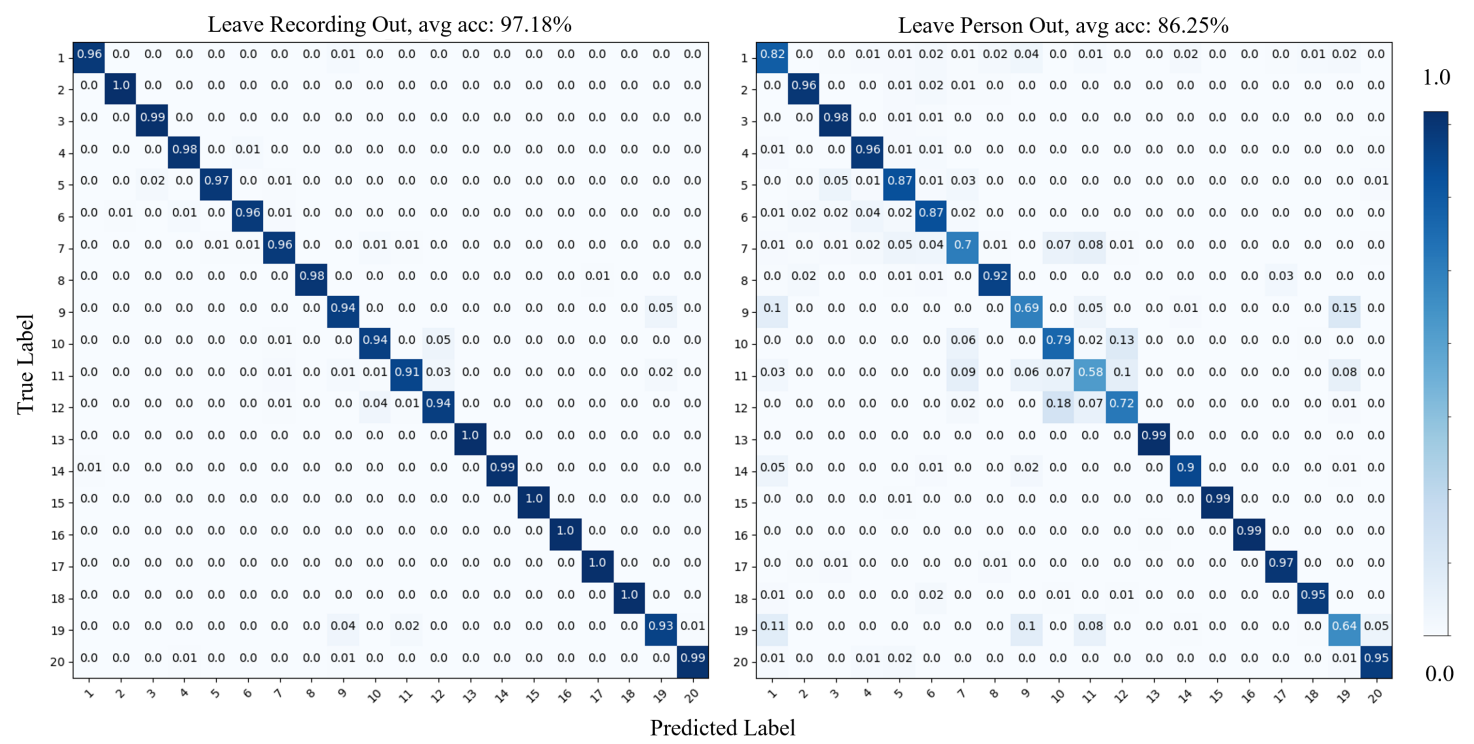

Figure 4: Confusion matrices for Conv2D. (left) Leave Recording Out, avg acc: $97.18 \%$, (right) Leave Person Out (stranger), avg acc: $86.25 \%$

for the second convolution (40,40). The third convolution is of size $(4,40)$ without pooling. A flattening layer of 160 is followed by a fully connected layer of 100 . The twenty outputs for the different activities in Figure 1 are then converted into probabilities by a fully connected layer and softmax function. The neural network is optimized by using the categorical cross-entropy loss function and Adam optimizer [26].

\subsection{Results and Discussion}

The results of three different models are shown in Table 2 and the confusion matrices for Conv2D in Figure 4. The classification results do not vary much across different models. Our method can robustly detect all 20 activities within a known group of 14 users from the LRO result with $95 \%$ average accuracy. With strangers (LPO), our method still yields on average above $85 \%$ accuracy; and nine classes out of the 20 return above $95 \%$ accuracy. Hence, our model works well for newly collected data from different people.

The LPO confusion matrix shows several pairs of significant misclassifications. We discuss the confusion matrix together with the postures and gestures and signal examples from Figure 1. For the pairs of arms-up (Gesture 12) / open-arms (10) forearms-block (9) / frame-picture (19), the arm motions and directions are physically similar. For the pair of lean-forward (1) / frame-picture (19), the signals look similar, which could be due to participants of different body shapes. However, the pair of forearms-block (9) / hands-onhead(11) also have similar motion (elbow flexion) and similar signal patterns, but with less misclassification. We can observe the activities involving shoulder movements, including shrug (7), forearmsblock (9), hands-on head (11), arms-up(12), frame-picture(19), all have apparent accuracy degradation compared to the LRO result. We suspect two factors cause this confusion: the MoCaBlazer does not have antennas covering the shoulder blades; our participants of different body sizes wore the same one-size blazer. Note that the experiment was in an office with few metallic objects nearby, which is known to affect capacitive sensing [37]. The impact of common and subtle disturbances was reduced by normalization of the BPG instances. Continuous recognition was performed as a "gesture spotting" validation. A sliding window of $2 \mathrm{~s}$ with a step of $0.5 \mathrm{~s}$ in an LPO scheme was deployed inside a 1D-LeNet5. We added the null class as the steady-state, for a total of 21 classes. Our results show $81.24 \pm 6.84$ accuracy and a weighted F1 score of $82.20 \pm 5.51$, suggesting that the system might be useful as a game controller.

\section{CONCLUSION AND OUTLOOK}

This paper has proposed an OpenTheremin-derived method to detect body postures and gestures with conductive textile as antennas that can be integrated into loose garments. We validated the proposed approach by the MoCaBlazer prototype produced through garment-sensing co-design with 14 diverse participants and a dictionary of 20 upper-body gestures. With the selected models such as 1D-LeNet5, DeepConvLSTM, and Conv2D, the system yielded competitive performance compared to state-of-the-art in loose garments for BPG detection. Moreover, the experimental results showed that the MoCaBlazer was robust against disturbances introduced by repeated wearing between each session ( 5 per volunteer). The noncontact capacitive sensing method has the advantages of being independent of muscular strength and avoidance of tight or flexible garments. In addition, it is relatively not sensitive to sweat or skin dryness [61]. However, the capacitive sensing method is susceptible to conductors, including persons/objects in close range with different dielectric properties compared to the antennas [37]. We normalized the data within each window to remove the absolute values during signal processing, and the deep learning models rely on the relative differences between channels. Thus, our approach is intrinsically robust against value drifting caused by floating grounds in capacitive sensing.

As our study has shown potential in loose garments, we would further pursue more elaborated garment integration; including miniaturized sensing modules, more channels, stretchable antennas, and sensor fusion with other modalities such as RFID to provide insitu re-calibration. We may also research creating a deep learning model that can reuse channel knowledge to adapt to more channels. 


\section{ACKNOWLEDGMENTS}

This work has been supported by BMBF (German Federal Ministry of Education and Research) in the project SocialWear.

\section{REFERENCES}

[1] Zamir Ahmed Abro, Yi-Fan Zhang, Cheng-Yu Hong, Rafique Ahmed Lakho, and Nan-Liang Chen. 2018. Development of a smart garment for monitoring body postures based on FBG and flex sensing technologies. Sensors and Actuators A: Physical 272 (2018), 153-160.

[2] Sizhen Bian, Vitor F Rey, Peter Hevesi, and Paul Lukowicz. 2019. Passive capacitive based approach for full body gym workout recognition and counting. In 2019 IEEE International Conference on Pervasive Computing and Communications (PerCom. IEEE, Kyoto, Japan, 1-10.

[3] Sizhen Bian, Vitor F Rey, Junaid Younas, and Paul Lukowicz. 2019. Wrist-Worn Capacitive Sensor for Activity and Physical Collaboration Recognition. In 2019 IEEE International Conference on Pervasive Computing and Communications Workshops (PerCom Workshops). IEEE, Kyoto, Japan, 261-266.

[4] Ali Boyali, Manolya Kavakli, et al. 2012. A robust and fast gesture recognition method for wearable sensing garments. In Proc. Int. Conf. Adv. Multimedia. International Academy, Research, and Industry Association (IARIA), Chamonix Mont Blanc, France, 142-147.

[5] Andreas Braun, Sebastian Frank, Martin Majewski, and Xiaofeng Wang. 2015 CapSeat: capacitive proximity sensing for automotive activity recognition. In Proceedings of the 7th International Conference on Automotive User Interfaces and Interactive Vehicular Applications. Association for Computing Machinery, Nottingham, United Kingdom, 225-232.

[6] Andreas Braun, Reiner Wichert, Arjan Kuijper, and Dieter W Fellner. 2015. Capacitive proximity sensing in smart environments. fournal of Ambient Intelligence and Smart Environments 7, 4 (2015), 483-510.

[7] Hammad Tanveer Butt, Manthan Pancholi, Mathias Musahl, Pramod Murthy, Maria Alejandra Sanchez, and Didier Stricker. 2019. Inertial Motion Capture Using Adaptive Sensor Fusion and Joint Angle Drift Correction. In 2019 22th International Conference on Information Fusion (FUSION). IEEE, Ottawa, ON, Canada, 1-8.

[8] Youngsu Cha, Hojoon Kim, and Doik Kim. 2018. Flexible piezoelectric sensorbased gait recognition. Sensors 18, 2 (2018), 468.

[9] Youngsu Cha, Kihyuk Nam, and Doik Kim. 2017. Patient posture monitoring system based on flexible sensors. Sensors 17, 3 (2017), 584

[10] Harish Chander, Reuben F Burch, Purva Talegaonkar, David Saucier, Tony Luczak, John E Ball, Alana Turner, Sachini NK Kodithuwakku Arachchige, Will Carroll, Brian K Smith, et al. 2020. Wearable stretch sensors for human movement monitoring and fall detection in ergonomics. International journal of environmental research and public health 17, 10 (2020), 3554.

[11] Jingyuan Cheng, Oliver Amft, Gernot Bahle, and Paul Lukowicz. 2013. Designing sensitive wearable capacitive sensors for activity recognition. IEEE sensors journal 13, 10 (2013), 3935-3947.

[12] Jingyuan Cheng, Oliver Amft, and Paul Lukowicz. 2010. Active capacitive sensing: Exploring a new wearable sensing modality for activity recognition. In International conference on pervasive computing. Springer, Berlin, Heidelberg, 319-336.

[13] Gabe Cohn, Sidhant Gupta, Tien-Jui Lee, Dan Morris, Joshua R Smith, Matthew S Reynolds, Desney S Tan, and Shwetak N Patel. 2012. An ultra-low-power human body motion sensor using static electric field sensing. In Proceedings of the 2012 ACM Conference on Ubiquitous Computing. Association for Computing Machinery, Pittsburgh, Pennsylvania, 99-102.

[14] Gabe Cohn, Daniel Morris, Shwetak Patel, and Desney Tan. 2012. Humantenna: using the body as an antenna for real-time whole-body interaction. In Proceedings of the SIGCHI Conference on Human Factors in Computing Systems. Association for Computing Machinery, Austin, Texas, USA, 1901-1910.

[15] Daniyar Enikeev and Svetlana Mustafina. 2020. Recognition of Sign Language Using Leap Motion Controller Data. In 2020 2nd International Conference on Control Systems, Mathematical Modeling, Automation and Energy Efficiency (SUMMA). IEEE, Lipetsk, Russia, 393-397.

[16] Urs Gaudenz. 2016. Open.Theremin V3, GaudiLabs. GaudiLabs LLC. Retrieved June 10, 2021 from http://www.gaudi.ch/OpenTheremin/index.php/opentheremin-v3

[17] Marian Haescher, Denys JC Matthies, Gerald Bieber, and Bodo Urban. 2015. Capwalk: A capacitive recognition of walking-based activities as a wearable assistive technology. In Proceedings of the 8th ACM International Conference on PErvasive Technologies Related to Assistive Environments. Association for Computing Machinery, Corfu, Greece, 1-8.

[18] Holger Harms, Oliver Amft, Gerhard Tröster, and Daniel Roggen. 2008. Smash: A distributed sensing and processing garment for the classification of upper body postures. In Proceedings of the ICST 3rd international conference on Body area networks. ICST (Institute for Computer Sciences, Social-Informatics and Telecommunications Engineering), Tempe, Arizona, 1-8.
[19] Paul Holleis, Albrecht Schmidt, Susanna Paasovaara, Arto Puikkonen, and Jonna Häkkilä. 2008. Evaluating capacitive touch input on clothes. In Proceedings of the 10th international conference on Human computer interaction with mobile devices and services. Association for Computing Machinery, Amsterdam, The Netherlands, 81-90.

[20] Sergey Ioffe and Christian Szegedy. 2015. Batch normalization: Accelerating deep network training by reducing internal covariate shift. In International conference on machine learning. PMLR, Lille, France, 448-456.

[21] Pyeong-Gook Jung, Gukchan Lim, Seonghyok Kim, and Kyoungchul Kong. 2015. A wearable gesture recognition device for detecting muscular activities based on air-pressure sensors. IEEE Transactions on Industrial Informatics 11, 2 (2015), 485-494.

[22] Julio Cezar Silveira Jacques Junior, Yağmur Güçlütürk, Marc Pérez, Umut Güçlü, Carlos Andujar, Xavier Baró, Hugo Jair Escalante, Isabelle Guyon, Marcel AJ Van Gerven, Rob Van Lier, et al. 2019. First impressions: A survey on vision-based apparent personality trait analysis. IEEE Transactions on Affective Computing 1, 1 (2019), 1-1.

[23] Muhammad US Khan, Assad Abbas, Mazhar Ali, Muhammad Jawad, and Samee U Khan. 2018. Convolutional neural networks as means to identify apposite sensor combination for human activity recognition. In 2018 IEEE/ACM International Conference on Connected Health: Applications, Systems and Engineering Technologies (CHASE). IEEE, 45-50.

[24] Muhammad US Khan, Assad Abbas, Mazhar Ali, Muhammad Jawad, and Samee U Khan. 2018. Convolutional neural networks as means to identify apposite sensor combination for human activity recognition. In 2018 IEEE/ACM International Conference on Connected Health: Applications, Systems and Engineering Technologies (CHASE). IEEE, 45-50.

[25] Ali Kiaghadi, Morgan Baima, Jeremy Gummeson, Trisha Andrew, and Deepak Ganesan. 2018. Fabric as a sensor: Towards unobtrusive sensing of human behavior with triboelectric textiles. In Proceedings of the 16th ACM Conference on Embedded Networked Sensor Systems. Association for Computing Machinery, Shenzhen, China, 199-210.

[26] Diederik P. Kingma and Jimmy Ba. 2017. Adam: A Method for Stochastic Optimization. arXiv:1412.6980 [cs.LG]

[27] Yuya Koyama, Michiko Nishiyama, and Kazuhiro Watanabe. 2016. Gait monitoring for human activity recognition using perceptive shoe based on hetero-core fiber optics. In 2016 IEEE 5th Global Conference on Consumer Electronics. IEEE, Kyoto, Japan, 1-2.

[28] Yuya Koyama, Michiko Nishiyama, and Kazuhiro Watanabe. 2018. Physical activity recognition using hetero-core optical fiber sensors embedded in a smart clothing. In 2018 IEEE 7th Global Conference on Consumer Electronics (GCCE). IEEE, Nara, Japan, 71-72.

[29] Arnaldo G Leal-Junior, Diana Ribeiro, Leticia M Avellar, Mariana Silveira, Camilo A Rodriguez Díaz, Anselmo Frizera-Neto, Wilfried Blanc, Eduardo Rocon, and Carlos Marques. 2020. Wearable and Fully-Portable Smart Garment for Mechanical Perturbation Detection With Nanoparticles Optical Fibers. IEEE Sensors fournal 21, 3 (2020), 2995-3003.

[30] Yann LeCun, Léon Bottou, Yoshua Bengio, and Patrick Haffner. 1998. Gradientbased learning applied to document recognition. Proc. IEEE 86, 11 (1998), 22782324.

[31] Junchan Li, Yu Wang, Pengfei Wang, Qing Bai, Yan Gao, Hongjuan Zhang, and Baoquan Jin. 2021. Pattern Recognition for Distributed Optical Fiber Vibration Sensing: A Review. IEEE Sensors fournal 21, 10 (2021), 11983-11998.

[32] Qi Lin, Shuhua Peng, Yuezhong Wu, Jun Liu, Wen Hu, Mahbub Hassan, Aruna Seneviratne, and Chun H Wang. 2020. E-Jacket: Posture Detection with LooseFitting Garment using a Novel Strain Sensor. In 2020 19th ACM/IEEE International Conference on Information Processing in Sensor Networks (IPSN). IEEE, Sydney, NSW, Australia, 49-60.

[33] Haoyan Liu, Enrique Sanchez, James Parkerson, and Alexander Nelson. 2019. Gesture Classification with Low-Cost Capacitive Sensor Array for Upper Extremity Rehabilitation. In 2019 IEEE SENSORS. IEEE, Montreal, QC, Canada, 1-4

[34] Gabriel Loke, Tural Khudiyev, Brian Wang, Stephanie Fu, Syamantak Payra, Yorai Shaoul, Johnny Fung, Ioannis Chatziveroglou, Pin-Wen Chou, Itamar Chinn, et al. 2021. Digital electronics in fibres enable fabric-based machine-learning inference. Nature communications 12, 1 (2021), 1-9.

[35] Mohammad Iman Mokhlespour Esfahani and Maury A Nussbaum. 2019. Classifying diverse physical activities using "Smart Garments". Sensors 19, 14 (2019), 3133.

[36] Francisco Javier Ordóñez and Daniel Roggen. 2016. Deep convolutional and lstm recurrent neural networks for multimodal wearable activity recognition. Sensors 16, 1 (2016), 115.

[37] Bryce Osoinach. 2007. Proximity capacitive sensor technology for touch sensing applications. Freescale White Paper 12 (2007).

[38] Arash Pouryazdan, Robert J Prance, Helen Prance, and Daniel Roggen. 2016. Wearable electric potential sensing: a new modality sensing hair touch and restless leg movement. In Proceedings of the 2016 ACM international joint conference on pervasive and ubiquitous computing: adjunct. Association for Computing Machinery, Heidelberg, Germany, 846-850. 
[39] Rajarajan Ramalingame, Rim Barioul, Xupeng Li, Giuseppe Sanseverino, Dominik Krumm, Stephan Odenwald, and Olfa Kanoun. 2021. Wearable Smart Band for American Sign Language Recognition with Polymer Carbon Nanocomposite based Pressure Sensors. IEEE Sensors Letters 5, 6 (2021), 6001204.

[40] Sanat Sarangi, Somya Sharma, and Bhushan Jagyasi. 2015. Agricultural activity recognition with smart-shirt and crop protocol. In 2015 IEEE Global Humanitarian Technology Conference (GHTC). IEEE, Seattle, WA, USA, 298-305.

[41] Sungtae Shin, Han Ul Yoon, and Byungseok Yoo. 2021. Hand Gesture Recognition Using EGaIn-Silicone Soft Sensors. Sensors 21, 9 (2021), 3204.

[42] Chamani Shiranthika, Nilantha Premakumara, Huei-Ling Chiu, Hooman Samani, Chathurangi Shyalika, and Chan-Yun Yang. 2020. Human Activity Recognition Using CNN \& LSTM. In 2020 5th International Conference on Information Technology Research (ICITR). IEEE, 1-6.

[43] Gurashish Singh, Alexander Nelson, Ryan Robucci, Chintan Patel, and Nilanjan Banerjee. 2015. Inviz: Low-power personalized gesture recognition using wearable textile capacitive sensor arrays. In 2015 IEEE international conference on pervasive computing and communications (PerCom). IEEE, St. Louis, MO, USA 198-206.

[44] Sophie Skach, Rebecca Stewart, and Patrick GT Healey. 2018. Smart arse: posture classification with textile sensors in trousers. In Proceedings of the 20th ACM International Conference on Multimodal Interaction. Association for Computing Machinery, Boulder, CO, USA, 116-124.

[45] Kenneth D Skeldon, Lindsay M Reid, Viviene McInally, Brendan Dougan, and Craig Fulton. 1998. Physics of the Theremin. American fournal of Physics 66, 11 (1998), 945-955.

[46] M Sornam, Kavitha Muthusubash, and V Vanitha. 2017. A survey on image classification and activity recognition using deep convolutional neural network architecture. In 2017 Ninth International Conference on Advanced Computing (ICoAC). IEEE, 121-126.

[47] Nitish Srivastava, Geoffrey Hinton, Alex Krizhevsky, Ilya Sutskever, and Ruslan Salakhutdinov. 2014. Dropout: a simple way to prevent neural networks from overfitting. The journal of machine learning research 15, 1 (2014), 1929-1958.

[48] Paul Stoffregen. 2014. FreqCount. Teensy. Retrieved June 10, 2021 from https: //www.pjrc.com/teensy/td_libs_FreqCount.html

[49] Paul Stoffregen. 2020. Teensy. Teensy. Retrieved June 10, 2021 from https: //www.pjrc.com/store/teensy41.html

[50] Saiganesh Swaminathan, Jonathon Fagert, Michael Rivera, Andrew Cao, Gierad Laput, Hae Young Noh, and Scott E Hudson. 2020. OptiStructures: Fabrication of Room-Scale Interactive Structures with Embedded Fiber Bragg Grating Optical Sensors and Displays. Proceedings of the ACM on Interactive, Mobile, Wearable and Ubiquitous Technologies 4, 2 (2020), 1-21.

[51] Nintendo Switch. 2020. Fust Dance. Nintendo. Retrieved August 05, 2021 from https://www.youtube.com/watch?v=_Fx-cnrNoM

[52] Liang Wang, Tao Gu, Xianping Tao, and Jian Lu. 2016. Toward a wearable RFID system for real-time activity recognition using radio patterns. IEEE Transactions on Mobile Computing 16, 1 (2016), 228-242.

[53] Interactive Wear. 2021. TWC24004B. Interactive Wear. Retrieved June 10, 2021 from http://www.interactive-wear.com/solutions/10-textile-wires/45textile-cables

[54] Nintendo Wii. 2009. Nintendo Wii's Rayman Raving Rabbids: TV Party - ShakeTV. Nintendo. Retrieved August 05, 2021 from https://www.youtube.com/watch?v= eoxjA6E1mDs

[55] Mathias Wilhelm, Daniel Krakowczyk, Frank Trollmann, and Sahin Albayrak. 2015. eRing: multiple finger gesture recognition with one ring using an electric field. In Proceedings of the 2nd international Workshop on Sensor-based Activity Recognition and Interaction. Association for Computing Machinery, Rostock, Germany, 1-6.

[56] Raphael Wimmer, Matthias Kranz, Sebastian Boring, and Albrecht Schmidt. 2007. Captable and capshelf-unobtrusive activity recognition using networked capacitive sensors. In 2007 Fourth International Conference on Networked Sensing Systems. IEEE, Braunschweig, Germany, 85-88.

[57] Yong Ye, Chiya Zhang, Chunlong He, Xi Wang, Jianjun Huang, and Jiahao Deng. 2020. A review on applications of capacitive displacement sensing for capacitive proximity sensor. IEEE Access 8 (2020), 45325-45342.

[58] Qihang Zeng, Wei Xu, Changyuan Yu, Na Zhang, and Cheungchuen Yu. 2018 Fiber-optic Activity Monitoring with Machine Learning. In Conference on Lasers and Electro-Optics/Pacific Rim. Optical Society of America, Hong Kong, China, W4K-5.

[59] Yang Zhang, Chouchang Yang, Scott E Hudson, Chris Harrison, and Alanson Sample. 2018. Wall++ room-scale interactive and context-aware sensing. In Proceedings of the 2018 CHI Conference on Human Factors in Computing Systems. Association for Computing Machinery, Montreal QC, Canada, 1-15.

[60] Zhen Zhang, Kuo Yang, Jinwu Qian, and Lunwei Zhang. 2019. Real-time surface EMG pattern recognition for hand gestures based on an artificial neural network. Sensors 19, 14 (2019), 3170.

[61] Enhao Zheng and Qining Wang. 2016. Noncontact capacitive sensing-based locomotion transition recognition for amputees with robotic transtibial prostheses. IEEE Transactions on Neural Systems and Rehabilitation Engineering 25, 2 (2016),
161-170.

[62] Bo Zhou, Mathias Sundholm, Jingyuan Cheng, Heber Cruz, and Paul Lukowicz. 2017. Measuring muscle activities during gym exercises with textile pressure mapping sensors. Pervasive and Mobile Computing 38 (2017), 331-345. 